Gazi University
Journal of Science
$\mathrm{http} / /$ dergipark.gov.tr/gujs

\title{
Some Identities of Harmonic and Hyperharmonic Fibonacci Numbers
}

\author{
Mirac CETIN ${ }^{1}$, Can KIZILATES ${ }^{2, *(1)}$, Fatma YESIL BARAN ${ }^{(1)}$, Naim TUGLU $^{4}$ \\ ${ }^{1}$ Baskent University, Department of Mathematics Education, 06790, Ankara, Turkey \\ ${ }^{2}$ Zonguldak Bulent Ecevit University, Department of Mathematics, 67100, Zonguldak, Turkey \\ ${ }^{3}$ Amasya University, Department of Computer Engineering, 05100, Amasya, Turkey \\ ${ }^{4}$ Gazi University, Department of Mathematics, 06500, Ankara, Turkey
}

Highlights

- This paper is deal with some identities of the harmonic and the hyperharmonic Fibonacci numbers.

- By using the symmetric algorithm, we get some important identities which improve the usual results.

- By using the Riordan array, some identities for hyperharmonic Fibonacci numbers are derived.

\section{Article Info}

Received:18 Mar 2020

Accepted: 16 Sep 2020

\section{Keywords}

Hyperharmonic

Fibonacci numbers,

Riordan array,

Symmetric

Infinite matrix method

\begin{abstract}
This paper is concerned with the combinatorial identities of the harmonic and the hyperharmonic Fibonacci numbers. By using the symmetric algorithm, we get some identities which improve the usual results and generalize known equations. Moreover, with the help of concept of Riordan array, we obtain the generating functions for these numbers and a variety of identities are derived.
\end{abstract}

\section{INTRODUCTION}

Harmonic numbers became popular in combinatorial problems and special functions. They are studied in many areas of mathematics and especially are considerable for number theory. They are related to harmonic series and Riemann zeta function. The $n$-th harmonic number, denoted by $H_{n}$, is defined by

$$
H_{n}=\sum_{k=1}^{n} \frac{1}{k}
$$

where $H_{0}=0$. Conway and Guy [1] generalized the harmonic numbers and they called the $n$-th hyperharmonic number of order $r$, denoted by $H_{n}^{(r)}$, as

$$
H_{n}^{(r)}=\sum_{k=1}^{n} H_{k}^{(r-1)} \quad(n, r \geq 1)
$$

where $H_{n}^{(0)}=\frac{1}{n}$ and $H_{n}^{(1)}=H_{n}$ is the $n$-th ordinary harmonic number. 
Fibonacci sequence is one of the most popular and useful sequence in mathematics. Therefore, this sequence has been investigated extensively. The Euler-Seidel matrix, which is given by [2], is useful to obtain some properties of the hyperharmonic and the $r$-Stirling numbers. Dil and Mezö [3] defined the hyper-Fibonacci numbers and the hyper-Lucas numbers. Hyper-Fibonacci numbers [3] is denoted by $F_{n}^{(r)}$, for any $r$ positive integer

$$
F_{n}^{(r)}=\sum_{k=1}^{n} F_{n}^{(r-1)} \quad(n, r \geq 1)
$$

with $F_{n}^{(0)}=F_{n}, F_{0}^{(r)}=0, F_{1}^{(r)}=1$. Also by using recurrence relation which define $a_{n}^{k}=a_{n-1}^{k}+a_{n}^{k-1}$, the authors introduce a symmetric algorithm. With the help of the symmetric algorithm, we can obtain some properties of the hyperharmonic numbers, Fibonacci and Lucas numbers and incomplete Fibonacci and Lucas numbers. Bahşi et al. [4] analyzed combinatorial properties of the hyper-Fibonacci and hyper-Lucas numbers by using a symmetric algorithm. Recently, in [5] Mezö and Ramirez obtained new algorithm which named the $q$-analogue of symmetric matrix algorithm. Moreover, the authors give several analytic and number theoretic identities for the $q$-hyperharmonic numbers. For more details about these algorithms see [6-8].

Fibonacci zeta function is defined by

$$
\zeta_{F}(s)=\sum_{k=1}^{\infty} \frac{1}{F_{k}^{s}}
$$

and some combinatorial properties of this function have been studied in $[9,10]$. But simple form of $\sum_{k=1}^{\infty} \frac{1}{F_{k}}$ for finite sums of reciprocals Fibonacci numbers is not known [11].

Tuglu et al. [12] called this finite sum as the $n$-th harmonic Fibonacci number by

$$
\mathbb{F}_{n}=\sum_{k=1}^{n} \frac{1}{F_{k}}
$$

and for any $r$ positive integer, they introduced the $n$-th hyperharmonic Fibonacci number, denoted by $\mathbb{F}_{n}^{(r)}$, by the undermentioned recurrence relations

$$
\mathbb{F}_{n}^{(r)}=\sum_{k=1}^{n} \mathbb{F}_{k}^{(r-1)} \quad(n, r \geq 1)
$$

with $\mathbb{F}_{n}^{(0)}=\frac{1}{\mathrm{~F}_{\mathrm{n}}}, \mathbb{F}_{0}^{(r)}=0$ and $\mathbb{F}_{0}=0$. For $r=1$, in particular,

$$
\mathbb{F}_{n}^{(1)}=\mathbb{F}_{n}=\sum_{k=1}^{n} \frac{1}{F_{k}}
$$

is obtained. Tuglu and Kızılateş [13-14] studied norms of some circulant and special matrices involving the harmonic Fibonacci and the hyperharmonic Fibonacci numbers.

Riordan matrices have been studied in some combinatorial problems and identities. Shapiro et al. [15] introduced Riordan group, which has element of Riordan matrices and defined conception of Riordan array. A Riordan array is defined by a pair of formal power series as follows: 


$$
R=(g(x), f(x))=\left[d_{n, k}\right]_{n, k \geq 0}
$$

with $f(0)=0, f^{\prime}(0) \neq 0$ and $g(0) \neq 0$ such that

$$
d_{n, k}=\left[x^{n}\right] g(x) f(x)^{k}
$$

where $\left[x^{n}\right]$ denotes the coefficient operator of $x^{n}$ obtained from $f(x)$. Using this definition, for any sequence $\left\{h_{k}\right\}$ having $h(x)$ as its generating function, we have

$$
\sum_{k=1}^{n} d_{n, k} h_{k}=\left[x^{n}\right] g(x) h(f(x)) .
$$

The above identity is named the fundamental theorem of Riordan arrays. The authors [16-18] studied the harmonic numbers with related to Riordan arrays.

In this study, our aim is to get unknown combinatorial properties of the hyperharmonic and the harmonic Fibonacci numbers. The paper is formed as follows. In section 2, we give the generating functions of the harmonic Fibonacci and hyperharmonic Fibonacci numbers respectively. In section 3, Mezö and Ramirez [5] obtained unknown number theoretic identities via $q$-harmonic numbers with help of $q$-symmetric algorithm. By using this algorithm, we give several combinatorial identities for the harmonic and hyperharmonic Fibonacci numbers which weren't available in literature. Finally, in section 4, with aid of concept of Riordan array, we call attention some formulas for these numbers.

\section{THE GENERATING FUNCTIONS}

A $q$-analogue of logarithm [19] is given by

$$
L_{\{q\}}(x)=\sum_{n=1}^{\infty} \frac{x^{n}}{q^{n}-1}, \quad|q|>1, \quad|x|<|q| .
$$

And Murty [20] showed that

$$
\sum_{n=1}^{\infty} \frac{1}{F_{n}}=(\alpha-\beta) L_{q}(-\alpha)
$$

with $q=-\alpha^{2}$ and $\alpha=\frac{1+\sqrt{5}}{2}, \beta=\frac{1-\sqrt{5}}{2}$.

The following theorem is about the generating function of $\mathbb{F}_{n}$.

Theorem 1. Let $\mathbb{F}_{n}$ be the $n$-th harmonic Fibonacci number. Then the generating function of $\mathbb{F}_{n}$ is

$$
\sum_{n=1}^{\infty} \mathbb{F}_{k} x^{n}=\frac{\alpha-\beta}{1-x} L_{-\alpha^{2}}(-\alpha x)
$$

where $L_{-\alpha^{2}}$ is as in (6).

Proof. Using the Equations (1), (6) and (7), we can obtain 


$$
\begin{aligned}
\sum_{n=1}^{\infty} \mathbb{F}_{n} x^{n} & =\sum_{n=1}^{\infty}\left(\sum_{k=1}^{n} \frac{1}{F_{k}}\right) x^{n} \\
& =\sum_{n=1}^{\infty}\left(\sum_{k=1}^{\infty} \frac{1}{F_{k}} x^{n+k}\right) \\
& =\sum_{n=1}^{\infty} x^{n} \sum_{k=1}^{\infty} \frac{1}{F_{k}} x^{k} \\
& =\frac{1}{1-x}(\alpha-\beta) \sum_{k=1}^{\infty} \frac{1}{\alpha^{k}-\beta^{k}} x^{k} \\
& =\frac{\alpha-\beta}{1-x} \sum_{k=1}^{\infty} \frac{(-\alpha)^{k}}{\left(-\alpha^{2}\right)^{k}-1} x^{k} \\
& =\frac{\alpha-\beta}{1-x} L_{-\alpha^{2}}(-\alpha x) .
\end{aligned}
$$

Corollary 1. Let $\mathbb{F}_{n}^{(r)}$ be the $n$-th hyperharmonic Fibonacci number. Then the generating function of $\mathbb{F}_{n}^{(r)}$ is

$$
\sum_{n=1}^{\infty} \mathbb{F}_{n}^{(r)} x^{n}=\frac{\alpha-\beta}{(1-x)^{r}} L_{-\alpha^{2}}(-\alpha x)
$$

Proof. Using the definition of the hyperharmonic Fibonacci numbers and the properties of generating functions, we get

$$
\begin{aligned}
\sum_{n=1}^{\infty} \mathbb{F}_{n}^{(r)} x^{n} & =\sum_{n=1}^{\infty}\left(\sum_{k_{r}=1}^{n} \ldots \sum_{k_{1}=1}^{k_{2}} \frac{1}{F_{k_{1}}}\right) x^{n} \\
& =\frac{1}{1-x}(\alpha-\beta) L_{-\alpha^{2}}(-\alpha x)
\end{aligned}
$$

which proves the corollary.

$$
\sum_{n=0}^{\infty} \mathbb{F}_{n+1}^{(r)} x^{n}=\frac{\alpha-\beta}{x(1-x)^{r}} L_{-\alpha^{2}}(-\alpha x) .
$$

Shifting indices for $n$ and using the Equation (9), the following equation is obtained

$$
\sum_{n=0}^{\infty} \mathbb{F}_{n+1}^{(r)} x^{n}=\frac{\alpha-\beta}{x(1-x)^{r}} L_{-\alpha^{2}}(-\alpha x) .
$$

\section{SYMMETRIC INFINITE MATRIX}

In this section, we obtain some identities involving the hyperharmonic Fibonacci numbers by using the symmetric infinite matrix.

Let $\left(a_{n}\right)$ and $\left(a^{n}\right)$ be sequences. The symmetric infinite matrix associated with these sequences fixed by the formulas

$$
a_{n}^{0}=a_{n}, a_{0}^{n}=a^{n} \quad(n \geq 0)
$$




$$
a_{n}^{k}=a_{n-1}^{k}+a_{n}^{k-1} \quad(n \geq 1, k \geq 1) .
$$

Let $a_{n}^{k}$ be any entries, where $k$ and $n$ are row and column respectively. Then using the symmetric relation $a_{n}^{k}$ can be obtained in terms of the first row's and the first column's elements as

$$
a_{n}^{k}=\sum_{i=1}^{k}\left(\begin{array}{c}
n+k-i-1 \\
n-1
\end{array}\right) a_{0}^{i}+\sum_{j=1}^{n}\left(\begin{array}{c}
k+n-j-1 \\
k-1
\end{array}\right) a_{j}^{0}
$$

(see [3] for more details).

Now we apply the symmetric infinite matrix method for $\mathbb{F}_{n}^{(r)}$ numbers. We start with $a_{n}^{0}=\mathbb{F}_{n+1}^{(m)}$ and $a_{0}^{n}=$ $\mathbb{F}_{1}^{(m+n)}=1(n \geq 1, m \geq 0)$ in (3.1). Then the following matrix

$$
\left(\begin{array}{lllll}
\mathbb{F}_{1}^{(m)} & \mathbb{F}_{2}^{(m)} & \mathbb{F}_{3}^{(m)} & \mathbb{F}_{4}^{(m)} & \cdots \\
\mathbb{F}_{1}^{(m+1)} & \mathbb{F}_{2}^{(m+1)} & \mathbb{F}_{3}^{(m+1)} & \mathbb{F}_{4}^{(m+1)} & \ldots \\
\mathbb{F}_{1}^{(m+2)} & \mathbb{F}_{2}^{(m+2)} & \mathbb{F}_{3}^{(m+2)} & \mathbb{F}_{4}^{(m+2)} & \ldots \\
\mathbb{F}_{1}^{(m+3)} & \mathbb{F}_{2}^{(m+3)} & \mathbb{F}_{3}^{(m+3)} & \mathbb{F}_{4}^{(m+3)} & \ldots \\
\vdots & \vdots & \vdots & \vdots & \ddots
\end{array}\right)
$$

is the corresponding infinite matrix.

Theorem 2. Let $n \geq 1, m \geq 0$ and $r>0$. We have

$$
\mathbb{F}_{n}^{(m+r)}=\sum_{s=1}^{n}\left(\begin{array}{c}
n+r-s-1 \\
r-1
\end{array}\right) \mathbb{F}_{s}^{(m)}
$$

Proof. Let us take $a_{n}^{0}=\mathbb{F}_{n+1}^{(m)}$ and $a_{0}^{n}=\mathbb{F}_{1}^{(m+n)}=1(n \geq 1)$ in (11). From (12), we obtain

$$
\begin{aligned}
a_{n+1}^{r+1} & =\sum_{i=1}^{r+1}\left(\begin{array}{c}
n+r-i+1 \\
n
\end{array}\right) a_{0}^{i}+\sum_{s=1}^{n+1}\left(\begin{array}{c}
n+r+1-s \\
r
\end{array}\right) a_{s}^{0} \\
& =\sum_{i=0}^{r}\left(\begin{array}{c}
n+r-i \\
n
\end{array}\right) a_{0}^{i+1}+\sum_{s=0}^{n}\left(\begin{array}{c}
n+r-s \\
r
\end{array}\right) a_{s+1}^{0} \\
& =\sum_{i=0}^{r}\left(\begin{array}{c}
n+r-i \\
n
\end{array}\right)+\sum_{s=0}^{n}\left(\begin{array}{c}
n+r-s \\
r
\end{array}\right) \mathbb{F}_{s+2}^{(m)}
\end{aligned}
$$

where $k=r-i$ and $b=n-s$. Using the equation $\sum_{t=a}^{c}\left(\begin{array}{l}t \\ a\end{array}\right)=\left(\begin{array}{c}c+1 \\ a+1\end{array}\right)$, we get

$$
\begin{aligned}
a_{n+1}^{r+1} & =\sum_{k=0}^{r}\left(\begin{array}{c}
n+k \\
n
\end{array}\right)+\sum_{b=0}^{n}\left(\begin{array}{c}
r+b \\
r
\end{array}\right) \mathbb{F}_{n-b+2}^{(m)} \\
& =\left(\begin{array}{c}
n+r+1 \\
n+1
\end{array}\right)+\sum_{b=0}^{n}\left(\begin{array}{c}
r+b \\
r
\end{array}\right) \mathbb{F}_{n-b+2}^{(m)} \\
& =\sum_{b=0}^{n+1}\left(\begin{array}{c}
r+b \\
r
\end{array}\right) \mathbb{F}_{n-b+2}^{(m)} .
\end{aligned}
$$

Shifting indices for $n$ and $r$, we obtain 


$$
\begin{aligned}
a_{n-1}^{r} & =\sum_{b=0}^{n-1}\left(\begin{array}{c}
r+b-1 \\
r-1
\end{array}\right) \mathbb{F}_{n-b}^{(m)} \\
& =\sum_{s=1}^{n}\left(\begin{array}{c}
n+r-s-1 \\
r-1
\end{array}\right) \mathbb{F}_{s}^{(m)} .
\end{aligned}
$$

From the definition of matrix in (13)

hence we have

$$
a_{n-1}^{r}=\mathbb{F}_{n}^{(m+r)}
$$

$$
\mathbb{F}_{n}^{(m+r)}=\sum_{s=1}^{n}\left(\begin{array}{c}
n+r-s-1 \\
r-1
\end{array}\right) \mathbb{F}_{s}^{(m)}
$$

We retrieve following corollary which is obtained in [12] by using the symmetric infinite matrix method.

Corollary 2. Let $\mathbb{F}_{n}^{(r)}$ be the $n$-th hyperharmonic Fibonacci number. Then the following equation hold

$$
\mathbb{F}_{n}^{(r)}=\sum_{s=1}^{n}\left(\begin{array}{c}
n+r-s-1 \\
r-1
\end{array}\right) \frac{1}{F_{s}}
$$

Proof. This is proved by setting $m=0$ in (14).

The following corollary is about the harmonic Fibonacci numbers.

Corollary 3. Let $\mathbb{F}_{n}$ be the harmonic Fibonacci number, and let $s$ be nonnegative number. Then

$$
\sum_{s=1}^{n}\left(\begin{array}{c}
n+r-s-1 \\
r-1
\end{array}\right) \mathbb{F}_{s}=\mathbb{F}_{n}^{(r+1)}
$$

Proof. The same proof remains valid for $m=1$ in Theorem 2 .

Corollary 4. For the hyperharmonic Fibonacci numbers, we have

$$
\sum_{s=1}^{n} s \mathbb{F}_{s}^{(m)}=n \mathbb{F}_{n}^{(m+1)}-\mathbb{F}_{n-1}^{(m+2)}
$$

Proof. Let $r=2$ in (14). We have

$$
\sum_{s=1}^{n}(n+1-s) \mathbb{F}_{s}^{(m)}=\mathbb{F}_{n}^{(m+2)}
$$

Then using the Equation (2), we get

$$
\sum_{s=1}^{n}(n+1-s) \mathbb{F}_{s}^{(m)}=(n+1) \sum_{s=1}^{n} \mathbb{F}_{s}^{(m)}-\sum_{s=1}^{n} s \mathbb{F}_{s}^{(m)}
$$




$$
=(n+1) \mathbb{F}_{n}^{(m+1)}-\sum_{s=1}^{n} s \mathbb{F}_{s}^{(m)} .
$$

Using (18) and recurrence relation for the hyperharmonic Fibonacci number (see Lemma 1 of Tuglu et al [12]), we obtain

$$
\begin{aligned}
\sum_{s=1}^{n} s \mathbb{F}_{s}^{(m)} & =(n+1) \mathbb{F}_{n}^{(m+1)}-\sum_{s=1}^{n}(n+1-s) \mathbb{F}_{s}^{(m)} \\
& =(n+1) \mathbb{F}_{n}^{(m+1)}-\mathbb{F}_{n}^{(m+2)} \\
& =(n+1) \mathbb{F}_{n}^{(m+1)}-\left(\mathbb{F}_{n-1}^{(m+2)}+\mathbb{F}_{n}^{(m+1)}\right) \\
& =n \mathbb{F}_{n}^{(m+1)}-\mathbb{F}_{n-1}^{(m+2)}
\end{aligned}
$$

which completes the proof.

Specially, if we take $m=1$ in (17), we get

$$
\sum_{k=1}^{n+1} k \mathbb{F}_{k}=(n+1) \mathbb{F}_{n+1}^{(2)}-\mathbb{F}_{n}^{(3)}
$$

Theorem 3. Let $\mathbb{F}_{n}^{(r)}$ be the $n$-th hyperharmonic Fibonacci number, and let $F_{n}$ be $n$-th Fibonacci number. Then

$$
\sum_{k=1}^{r} \mathbb{F}_{n}^{(k)}=\mathbb{F}_{n+1}^{(r)}-\frac{1}{F_{n+1}}
$$

Proof. By considering the Equation (15), we have

$$
\begin{aligned}
\sum_{k=1}^{r} \mathbb{F}_{n}^{(k)} & =\sum_{k=1}^{r} \sum_{t=1}^{n}\left(\begin{array}{c}
n+k-t-1 \\
k-1
\end{array}\right) \frac{1}{F_{t}} \\
& =\sum_{t=1}^{n} \frac{1}{F_{t}} \sum_{k=1}^{r}\left(\begin{array}{c}
n+k-t-1 \\
k-1
\end{array}\right) \\
& =\sum_{t=1}^{n}\left(\begin{array}{c}
n+r-t \\
r-1
\end{array}\right) \frac{1}{F_{t}} \\
& =\sum_{t=1}^{n+1}\left(\begin{array}{c}
n+r-t \\
r-1
\end{array}\right) \frac{1}{F_{t}}-\frac{1}{F_{n+1}} \\
& =\mathbb{F}_{n+1}^{(r)}-\frac{1}{F_{n+1}}
\end{aligned}
$$

which completes the proof.

\section{RIORDAN ARRAYS}

In this section, observing the triangle which is obtained from $\left(\mathbb{F}_{n}^{(r)}\right)$ given by (2) holds a Riordan array, we get some properties of $\left(\mathbb{F}_{n}^{(r)}\right)$. Also, we indicate how $\left(\mathbb{F}_{n}^{(r)}\right)$ numbers may be represented with aid of Riordan array. 
Lemma 1. Let $\mathbb{F}_{n}^{(r)}$ be the $n$-th hyperharmonic Fibonacci number, and let $\mathcal{F}=\left[f_{n, k}\right]_{n, k \geq 0}$ be infinite matrix, defined by

$$
f_{n, k}=\left\{\begin{array}{cc}
\mathbb{F}_{n-k+1}^{(r)} & n-k+1 \geq 0 \\
0 & n-k+1<0
\end{array}\right.
$$

The Riordan representation of $\mathcal{F}$ is

$$
\mathcal{F}=\left(\frac{\alpha-\beta}{x(1-x)^{r}} L_{-\alpha^{2}}(-\alpha x), x\right)
$$

Proof. The matrix $\mathcal{F}$ can be written as

$$
\left(\begin{array}{lllll}
\mathbb{F}_{1}^{(r)} & 0 & 0 & 0 & \cdots \\
\mathbb{F}_{2}^{(r)} & \mathbb{F}_{1}^{(r)} & 0 & 0 & \ldots \\
\mathbb{F}_{3}^{(r)} & \mathbb{F}_{2}^{(r)} & \mathbb{F}_{1}^{(r)} & 0 & \ldots \\
\mathbb{F}_{4}^{(r)} & \mathbb{F}_{3}^{(r)} & \mathbb{F}_{2}^{(r)} & \mathbb{F}_{1}^{(r)} & \ldots \\
\vdots & \vdots & \vdots & \vdots & \ddots
\end{array}\right)
$$

Using (10), we can see that $0^{\text {th }}$ column is accounted for coefficients $\frac{\alpha-\beta}{x(1-x)^{r}} L_{-\alpha^{2}}(-\alpha x)$. Then from the rule of Riordan group, we get Riordan representation of this matrix

$$
\left(\frac{\alpha-\beta}{x(1-x)^{r}} L_{-\alpha^{2}}(-\alpha x), x\right)
$$

which proves the lemma.

The Equation (14) can be proved by another way by using the Riordan array.

Another Proof. Let $h(x)=\frac{1}{(1-x)^{s}}=\sum_{n=0}^{\infty}\left(\begin{array}{c}s+n-1 \\ s-1\end{array}\right) x^{n}$. From (5) and (21), we have

$$
\begin{aligned}
\sum_{k=0}^{n}\left(\begin{array}{c}
s+k-1 \\
s-1
\end{array}\right) \mathbb{F}_{n-k+1}^{(r)} & =\left[x^{n}\right] \frac{\alpha-\beta}{x(1-x)^{r}} L_{-\alpha^{2}}(-\alpha x) \frac{1}{(1-x)^{s}} \\
& =\left[x^{n}\right] \frac{\alpha-\beta}{x(1-x)^{r+s}} L_{-\alpha^{2}}(-\alpha x) \\
& =\left[x^{n}\right] \sum_{k=0}^{\infty} \mathbb{F}_{n+1}^{(r+s)} x^{n} \\
& =\mathbb{F}_{n+1}^{(r+s)} .
\end{aligned}
$$

Also we have

$$
\sum_{k=0}^{n}\left(\begin{array}{c}
s+k-1 \\
s-1
\end{array}\right) \mathbb{F}_{n-k+1}^{(r)}=\sum_{k=1}^{n+1}\left(\begin{array}{c}
s+n-k \\
s-1
\end{array}\right) \mathbb{F}_{k}^{(r)}
$$

then we obtain

$$
\sum_{k=1}^{n+1}\left(\begin{array}{c}
s+n-k \\
s-1
\end{array}\right) \mathbb{F}_{n}^{(r)}=\mathbb{F}_{n+1}^{(r+s)}
$$




\section{Lemma 2. Let}

$$
\mathcal{F}=\left(\frac{\alpha-\beta}{x(1-x)} L_{-\alpha^{2}}(-\alpha x), \frac{x}{1-x}\right)
$$

be a Riordan matrix. The generic element $f_{n, k}$ of $\mathcal{F}$ is $\mathbb{F}_{n-k+1}^{(k+1)}$.

Proof. From (4) and (10), we get

$$
\begin{aligned}
f_{n, k} & =\left[x^{n}\right] \frac{\alpha-\beta}{x(1-x)} L_{-\alpha^{2}}(-\alpha x)\left(\frac{x}{1-x}\right)^{k} \\
& =\left[x^{n}\right] \frac{\alpha-\beta}{x(1-x)^{k+1}} L_{-\alpha^{2}}(-\alpha x) x^{k} \\
& =\left[x^{n-k}\right] \frac{\alpha-\beta}{x(1-x)^{k+1}} L_{-\alpha^{2}}(-\alpha x) \\
& =\left[x^{n-k}\right] \sum_{k=0}^{\infty} \mathbb{F}_{n+1}^{(k+1)} x^{n} \\
& =\mathbb{F}_{n-k+1}^{(k+1)} .
\end{aligned}
$$

Theorem 4. Let $\mathbb{F}_{n}^{(r)}$ be the $n$-th hyperharmonic Fibonacci number. We have

$$
\sum_{k=1}^{n+1}(-1)^{n-k+1} \mathbb{F}_{k}^{(n-k+2)}=\frac{1}{F_{n+1}}
$$

Proof. Let $h(x)=\frac{1}{1+x}=\sum_{n=0}^{\infty}(-1)^{n} x^{n}$. From (5), (7) and (22), we obtain

$$
\begin{aligned}
\sum_{k=0}^{n}(-1)^{k} \mathbb{F}_{n-k+1}^{(k+1)} & =\left[x^{n}\right] \frac{\alpha-\beta}{x(1-x)} L_{-\alpha^{2}}(-\alpha x) h\left(\frac{x}{1-x}\right) \\
& =\left[x^{n}\right] \frac{\alpha-\beta}{x(1-x)} L_{-\alpha^{2}}(-\alpha x)(1-x) \\
& =\left[x^{n}\right] \frac{\alpha-\beta}{x} L_{-\alpha^{2}}(-\alpha x) \\
& =\left[x^{n}\right] \sum_{n=0}^{\infty} \frac{1}{F_{n+1}} x^{n} \\
& =\frac{1}{F_{n+1}}
\end{aligned}
$$

And

$$
\sum_{k=0}^{n}(-1)^{k} \mathbb{F}_{n-k+1}^{(k+1)}=\sum_{k=1}^{n+1}(-1)^{n-k+1} \mathbb{F}_{k}^{(n-k+2)}
$$

So, we have

$$
\sum_{k=1}^{n+1}(-1)^{n-k+1} \mathbb{F}_{k}^{(n-k+2)}=\frac{1}{F_{n+1}}
$$


Theorem 5. Let $\mathbb{F}_{n}^{(r)}$ be the $n$-th hyperharmonic Fibonacci number, and let $s$ be nonnegative number. Then

$$
\sum_{k=1}^{n+1}\left(\begin{array}{c}
s \\
n-k+1
\end{array}\right) \mathbb{F}_{k}^{(n-k+2)}=\mathbb{F}_{n+1}^{(s+1)}
$$

Proof. Let $h(x)=(1+x)^{s}=\sum_{n=0}^{\infty}\left(\begin{array}{l}s \\ n\end{array}\right) x^{n}$. From (5) and (22), we have

$$
\begin{aligned}
\sum_{k=0}^{n}\left(\begin{array}{l}
s \\
k
\end{array}\right) \mathbb{F}_{n-k+1}^{(k+1)} & =\left[x^{n}\right] \frac{\alpha-\beta}{x(1-x)} L_{-\alpha^{2}}(-\alpha x) h\left(\frac{x}{1-x}\right) \\
& =\left[x^{n}\right] \frac{\alpha-\beta}{x(1-x)} L_{-\alpha^{2}}(-\alpha x) \frac{1}{(1-x)^{s}} \\
& =\left[x^{n}\right] \frac{\alpha-\beta}{x(1-x)^{s+1}} L_{-\alpha^{2}}(-\alpha x) \\
& =\left[x^{n}\right] \sum_{n=0}^{\infty} \mathbb{F}_{n+1}^{(s+1)} x^{n} \\
& =\mathbb{F}_{n+1}^{(s+1)} .
\end{aligned}
$$

Also, we get

$$
\sum_{k=0}^{n}\left(\begin{array}{l}
s \\
k
\end{array}\right) \mathbb{F}_{n-k+1}^{(k+1)}=\sum_{k=1}^{n+1}\left(\begin{array}{c}
s \\
n-k+1
\end{array}\right) \mathbb{F}_{k}^{(n-k+2)}
$$

Finally, we obtain that

$$
\sum_{k=1}^{n+1}\left(\begin{array}{c}
s \\
n-k+1
\end{array}\right) \mathbb{F}_{k}^{(n-k+2)}=\mathbb{F}_{n+1}^{(s+1)}
$$

Corollary 5. For the hyperharmonic Fibonacci numbers, we get

$$
\sum_{k=1}^{n+1}\left(\begin{array}{c}
n \\
k-1
\end{array}\right) \mathbb{F}_{k}^{(n-k+2)}=\mathbb{F}_{n+1}^{(n+1)}
$$

Proof. It can be simply seen that taking $s=n$ in (24).

\section{CONFLICTS OF INTEREST}

No conflict of interest was declared by the authors.

\section{REFERENCES}

[1] Conway, J. H., Guy, R. K., “The book of numbers”, Springer, New York, (1996).

[2] Dumont, D., “Matrices d'Euler-Seidel”, Semin. Lothar. Comb., 5 (1981). 
[3] Dil, A., Mezö, I., “A symmetric algorithm for hyperharmonic and Fibonacci numbers”, Appl. Math. Comput., 206: 942-951, (2008).

[4] Bahşi, M., Mezö, I., Solak, S., "A symmetric algorithm for hyper-Fibonacci and hyper-Lucas numbers", Ann. Math. Inform., 43:19-27, (2014).

[5] Mezö, I., Ramirez, J. L., “A q-Symmetric algorithm and its applications to some combinatorial sequences", Online J. Anal. Comb., 12: 1-13, (2017).

[6] Dil, A., Kurt, V., "Investigating geometric and exponential polynomials with Euler-Seidel matrices", J. Integer Seq., 14: 1-12, (2011).

[7] Dil, A., Mezö, I., "Euler-Seidel method for certain combinatorial numbers and a new characterization of Fibonacci sequence”, Cent. Eur. J. Math., 7(2): 310-321, (2009).

[8] Ramirez, J. L., Shattuck, M., “A multi-parameter generalization of the symmetric algorithm”, Math. Slovaca, 68(4): 699-712, (2018).

[9] Holliday, S., Komatsu, T., "On the sum of reciprocal generalized Fibonacci numbers", Integers, 11: 441-455, (2011).

[10] Ohtsuka, H., Nakamura, S., "On the sum of reciprocal Fibonacci numbers”, Fibonacci Quart., 46 (2):153-159, (2008).

[11] Rabinowitz, S., "Algorithmic summation reciprocals of products of Fibonacci numbers", Fibonacci Quart., 37: 122-127, (1999).

[12] Tuglu, N., Kızılateş, C., Kesim, S., "On the harmonic and hyperharmonic Fibonacci numbers”, Adv. Difference Equ., 2015: 297, (2015).

[13] Tuglu, N., Kızılateş C., "On the norms of circulant and r -circulant matrices with the hyperharmonic Fibonacci numbers", J.Inequal. Appl., 2015: 253, (2015).

[14] Tuglu, N., Kızılateş, C., "On the norms of some special matrices with the harmonic Fibonacci numbers", Gazi University Journal of Science, 28 (3): 447-501, (2015).

[15] Shapiro, L. Getu, W. S., Woan, W. J., Woodson, L. C., “The Riordan group”, Discrete Appl. Math., 34: 229-239, (1991).

[16] Cheon, G. S., Hwang, S. G., Lee, S. G., "Several polynomials associated with the harmonic numbers”, Discrete Appl.Math., 155: 2573-2584, (2007).

[17] Cheon, G. S., Mikkawy, M. E. A., "Generalized harmonic numbers with Riordan arrays", J. Number Theory, 128: 413-425, (2008).

[18] Munarini, E., "Riordan matrices and sums of harmonic numbers", Appl. Anal. Discrete Math., 5: 176-200, (2011). 
[19] Matala-Aho, T. and Vaananen, K., "On Approximation Measures of $q$-Logarithms”, Bull. Austral. Math. Soc., 58: 15-31, (1998).

[20] Murty, M. R., "The Fibonacci Zeta Function, Automorphic Representations and L-Functions”, Tata Institue of Fundamental Research, Hindustan Book Agency, New Delhi, 1-17, (2013). 